of practitioners not engaging in the study. 159 of the remaining 201 patients had the appropriate proforma section completed.

Results 125/159 (78.6\%) had performed unprotected sexual intercourse (UPSI) with their MRSP. 120/159 (75.4\%) stated that they knew whether or not their MRSP was using contraception. Of the 39 (24.6\%) who didn't know, 10 had performed only protected sex with their MRSP and a further two had vasectomies. Of the remaining 27 (17\%), 19/27 (70.4\%) classed their partner as "Casual" and 8/27 (29.6\%) "Regular". 19/37 (51.3\%) men having UPSI with a casual partner didn't know if they were on contraception.

Discussion/conclusion Poor knowledge of partner's contraceptive status is demonstrated. This may highlight a potential area for future intervention.

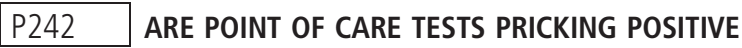 PEOPLE?}

Ryan McCloskey*, Mark Lawton. Royal Liverpool and Broadgreen University Hospitals, Liverpool, UK

\subsection{6/sextrans-2016-052718.288}

Background/introduction/Aims To establish whether point of care testing is being carried out in the appropriate populations. We endeavoured to see if our rapid HIV testing met the guideline specificity of $99.4 \%$

Methods We carried out a retrospective analysis of 674 patients who received point of care tests (POCTs) after attending the sexual health clinic at the Royal Liverpool Hospital between October 2014 and May 2015. We reviewed the point of care tests based upon the indication, age and sex of the patient. The outcome measures included whether the test was reactive or not and the final result of the laboratory HIV test. Indications were defined by individual staff members logging the information and if multiple indications were given, the aspect deemed to be higher risk was used

Results Of the 674 patients 499 were female and 175 were male. The four most common indications were for testing were MSM (36\%), Worried/Anxious (15\%), PEP (11.5\%) and HIV positive partner (9\%). 31 tests (4.8\%) were reactive and 23 tests (3.4\%) produced a corresponding positive final laboratory result. This equated to a specificity of $98.77 \%$. Positivity of indications varied remarkably-MSM, 3.7\% versus Worried,0\%.

Discussion/conclusion The study developed our understanding of the uptake of POCTs. A larger sample could have increased the specificity to the national guidance. The variability in documentation made it difficult to categorise risk in different indications. For example, indications of "high risk" and "worried" are vague and potentially misleading.

\section{P243 CHARACTERISING NEW HIV DIAGNOSES IN NORTH WEST NORTHERN IRELAND}

Alan Convery, Melissa Perry*. Western Health and Social Care Trust., Northern Ireland, UK

\subsection{6/sextrans-2016-052718.289}

Background/introduction North West Northern Ireland is a predominately rural population. New HIV diagnoses in Northern Ireland are rising.

Aim We sought to characterise the new diagnoses being made in this region.
Methods The service became a consultant-led service on a daily basis in November 2015 and since this date has been offering ongoing care for PLWH. We analysised demographic details of new HIV diagnoses including sex, age and sexual identity. Details regarding the health location of the first positive test and surrogate markers at diagnosis were also recorded.

Results Since November 2015 there have been 7 new HIV diagnoses. Two tested positive within the GU service; external referrals with a new positive test included 1 from General Practice, 1 from Oral Surgery and 3 from Gastroenterology. Of the 7 new diagnoses 6 were in men. All were of white Northern Irish ethnicity. 3 men were MSM and 3 were heterosexual. 6 of the 7 are thought to have acquired their HIV within NI. Age range at diagnosis was 27-64 years old, but 6/7 were 44 years old or over. One patient disengaged from care following diagnosis and no surrogate markers were obtained as a result of patient's refusal for further blood tests. Of the 6 that have remained in care only 1 patient had a baseline CD4 count of greater than 350 cells $/ \mathrm{mm}^{3} ; 2$ patients had a CD4 $<200$ cells $/ \mathrm{mm}^{3}$ at diagnosis.

Conclusion Although small numbers so far, this analysis suggests that new HIV diagnoses in this region are of an older age with advanced HIV.

\section{P244 HSV SEROTYPES IN FIRST EPISODE GENITAL HERPES IN NORTH WEST NORTHERN IRELAND}

Lynsey Melaugh, Melissa Perry*. Western Health and Social Care Trust., Northern Ireland, UK

\subsection{6/sextrans-2016-052718.290}

Background/introduction Most populations have observed an increase in the role of HSV-1 as a cause of genital herpes simplex virus (HSV)

Aims To analyse HSV serotypes in first episode genital herpes in North West Northern Ireland.

Methods All patients who tested positive for HSV and were coded as C10A from 01/03/2015 - 01/03/2016 were included. Demographic details including sex, age, ethnicity and sexual identity were recorded. The confirmed serotype (HSV-1 vs HSV2) was noted.

Results A total of 36 cases of first episode genital HSV was seen. $100 \%$ were white Caucasian (35/36 White UK); 29 were female and 7 were male. All females self-reported as heterosexual and of the 2/7 men were MSM. Overall median age at diagnosis was 23 years old (range 16-40). Of the 36 positive PCR tests $83.3 \%$ (30) were HSV-1 and 6.7\% (6) were HSV-2. The median age at diagnosis for HSV-1 was also 23 years (range 16-32); 26 were female and 4 male. Of the 6 positive who tested positive for HSV-2, 3 each were male and female.

Conclusion It is well documented that HSV-1 is increasingly prevalent as a cause of first episode genital HSV, our rate of $83 \%$ for HSV-1 infection is notably high. Although not a significant diagnostic or management challenge this may reflect a lower prevalence of HSV-2 infection within the local sexuallyactive population or an increasing level of sexual risk behaviour capable of transmitting HSV-1 infection. Local clinicians need to be aware of the differences in prognosis for HSV-1 infection such that accurate information and advice can be given to help reduce stigma traditionally attached to HSV-2 infection. 